\title{
COHOMOLOGY OF COXETER GROUPS AND ARTIN GROUPS
}

\author{
C. De Concini and M. Salvetti
}

\begin{abstract}
Given a finite Coxeter system $(\mathbf{W}, S)$, we exhibit an explicit "small" resolution of the trivial $\mathbb{Z}[\mathbf{W}]$-module. This is obtained by an explicit combinatorial construction of $k(\mathbf{W}, 1)$, which is described as a CW-complex with cells corresponding to flags in $S$. Some applications are given.
\end{abstract}

\section{Introduction}

Let $(\mathbf{W}, S)$ be an irreducible Coxeter system (with finite $\mathbf{W}$ ). In this paper we give the construction of an explicit free resolution $\left(\mathcal{C}_{*}, \delta_{*}\right)$ of the trivial $\mathbb{Z}[\mathbf{W}]$ module $\mathbb{Z}$. In dimension $k, \mathcal{C}_{k}$ is the free $\mathbb{Z}[\mathbf{W}]$ module with basis elements $e(\Gamma)$, $\Gamma \in S_{k}$, where

$$
S_{k}:=\left\{\Gamma=\left(\Gamma_{1} \supset \Gamma_{2} \supset \ldots\right)\left|\Gamma_{1} \subset S, \sum_{i \geq 1}\right| \Gamma_{i} \mid=k\right\}
$$

are the flags of subsets of $S$ of cardinality $k$. The $\mathbb{Z}[\mathbf{W}]$-linear differential $\delta_{k}: C_{k} \rightarrow C_{k-1}$ involves minimal coset representatives of parabolic subgroups of $\mathbf{W}([\mathrm{Bou}, \mathrm{Hum}])$. One has:

$$
\delta_{k} e(\Gamma)=\sum_{\substack{i \geq 1 \\\left|\Gamma_{i}\right|>\left|\Gamma_{i+1}\right|}} \sum_{\tau \in \Gamma_{i}} \sum_{\substack{\beta \in \mathbf{W}_{\Gamma_{i}}^{\Gamma_{i} \backslash\{\tau\}} \\ \beta^{-1} \Gamma_{i+1} \beta \subset \Gamma_{i} \backslash\{\tau\}}}(-1)^{\alpha(\Gamma, i, \tau, \beta)} \beta e\left(\Gamma^{\prime}\right) .
$$

Here

$$
\Gamma^{\prime}:=\left(\Gamma_{1} \supset \cdots \supset \Gamma_{i-1} \supset\left(\Gamma_{i} \backslash\{\tau\}\right) \supset \beta^{-1} \Gamma_{i+1} \beta \supset \beta^{-1} \Gamma_{i+2} \beta \supset \ldots\right) ;
$$

$\mathbf{W}_{\Gamma_{i}}^{\Gamma_{i} \backslash\{\tau\}}$ is the set of minimal coset representatives of the parabolic subgroup $\mathbf{W}_{\Gamma_{i} \backslash\{\tau\}}$ in $\mathbf{W}_{\Gamma_{i}}$; the exponent $\alpha$ is easily computed in terms of $\Gamma, \tau, \beta$ (see formula (11)).

Our resolution is much smaller than the so called standard resolution: in fact, $\left|S_{k}\right|=\left(\begin{array}{c}n+k-1 \\ k\end{array}\right)$, where $n=\operatorname{rank}(\mathbf{W})$, so the growth of the complex is

Received June 2, 1999. 
polynomial in $k$, while the standard one grows as $|\mathbf{W}|^{k}$ (we "conjecture" that our resolution is the smallest possible, among those which coincide with our up to dimension 2 ; see $[\mathrm{Sw}]$ for a different periodic resolution in case $A_{2}$ ).

Of course, by standard definitions, one can compute homology and cohomology of $\mathbf{W}$ with coefficients in any $\mathbf{W}$-module by using this resolution.

The previous results are obtained through a good topological description of $k(\mathbf{W}, 1)$. Actually, our methods generalize a series of previous papers [S0], [S1], [D-S]. In [D-S], we found algebraic complexes which compute the cohomology of the associated Artin group $\mathbf{G}_{\mathbf{W}}$ of type $\mathbf{W}$ in any local system of coefficients. In some sense, the Artin group $\mathbf{G}_{\mathbf{W}}$ has to be considered as a first order approximation of $\mathbf{W}$ : in fact, one has a natural embedding $k\left(\mathbf{G}_{\mathbf{W}}, 1\right) \hookrightarrow k(\mathbf{W}, 1)$ which corresponds to flags whose terms different from the first one are empty. The coboundary formula which we derive for Coxeter groups are similar to those for Artin groups, but more complicated. As it appears from above formula, at level $i$ one has to consider only minimal coset representatives such that conjugation of the $(i+1)$-th term of the flag is contained in the $i$-th term.

In the first part we introduce, given a vector space $M \cong \mathbb{R}^{d+1}$, the notion of $M$ (or $d+1$ )-complexification of a hyperplane $H \subset \mathbb{R}^{N}$ : it is the $d+1$-codimensional subspace in $\left(\mathbb{R}^{N}\right)^{d+1}:=\mathbb{R}^{N} \otimes M$ given by the same equation as $H$ in each factor (for $d=1$ it coincides with the standard complexification). Given an arrangement of hyperplanes in $\mathbb{R}^{N}$, we consider its $d+1$-complexification: it is possible to generalize [S0] and describe the homotopy type of the complement through a regular $C W$-complex. We find convenient to consider a suitable stratification of $\mathbb{R}^{N} \otimes M$, which generalizes those used in [G-R], [B-Z] for $d=1$. This generalization to subspaces is very natural and rather straightforward (similar considerations appear already in [B-Z; 9.3]). For completeness, and since in order to produce the above algebraic complex, we need a very precise description of the CW-complex in case of complexified subspaces, we prefer to re-prove everything in this case. Strata correspond bijectively to $d+1$-chains $F=\left(F_{1} \succ \ldots \succ F_{d+1}\right)$, where $F_{i}$ is a facet in the stratification of $\mathbb{R}^{N}$ induced by the arrangement. One orders those chains according to the reverse inclusion of the closure of the corresponding strata. The complement $\mathbf{Y}^{(d+1)}$ to the $(d+1)$-complexified family contracts over a $C W$-complex $\mathbf{X}^{(d)}$ which is the dual to the subposet given by those chains $F$ such that $F_{d+1}$ is a chamber.

In the second part we consider the case of an arrangement associated to a finite group $\mathbf{W}$ generated by reflections. In this case we describe a construction which generalizes [S1]. The orbit space $\mathbf{Y}^{(d+1)} / \mathbf{W}$ contracts over a $C W$-complex $\mathbf{X}_{\mathbf{W}}^{(d)}$ whose $k$-cells corresponds to the $d$-chains $\Gamma_{1} \supset \cdots \supset \Gamma_{d}$, where each $\Gamma_{i}$ is a subset of the set of standard generators $S$ of $W$ given by the reflections with respect to the walls of a fixed chamber, and $\sum_{i}\left|\Gamma_{i}\right|=k$.

Then in the third part we proceed to find an algebraic complex whose homology equals the integral homology of $\mathbf{X}^{(d)}$. The key step here is the algebraic characterization of cells with nonvanishing incidence (Theorem 3.1.3). The orientation of cells is induced by suitable differentiable forms. Since $\mathbf{X}^{(d+1)}$ is 
obtained from $\mathbf{X}^{(d)}$ by attaching cells of dimension $\geq d+1$, passing to the limit we get the above resolution. In fact, the space $k(\mathbf{W}, 1)$ is given by

$$
\lim _{d \rightarrow \infty} \mathbf{Y}^{(d)} / \mathbf{W} \cong \lim _{d \rightarrow \infty} \mathbf{X}^{(d)} / \mathbf{W}
$$

and $\mathbf{W}$ acts cellularely and freely over $\mathbf{X}^{(\infty)}:=\lim _{d \rightarrow \infty} \mathbf{X}^{(d)}$.

We consider finite Coxeter systems only for sake of simplicity. In fact, we remark here that all the previous results can be extended with slight modifications to any finitely generated Coxeter system: for example, in general one will have to consider flags of subsets not of all $S$, but of some subsets of $S$. The proofs are essentially the same.

In part 4 we give a geometric application. Let $p: \mathbf{Y}^{(2)} \rightarrow \mathbf{Y}^{(2)} / \mathbf{W}$ be the regular covering with base space $k\left(\mathbf{G}_{\mathbf{W}}, 1\right)$. We prove that the Schwartz genus of $p$ is equal to $\mathrm{rk}(\mathbf{W})+1$ for all irreducible Coxeter groups except in case $A_{n}$ when $n+1$ is not a prime power. In the latter case this result is still conjecturally true. Our result in the $A_{n}$ case has already been proved in [Va], where the author used specific methods for this situation: a stratification (which turns out to be equivalent to ours in this case) and cohomology with constant coefficients and with coefficients in the sign representation, with ad hoc formulas. The generalization (theorem 4.2.1) is a straightforward consequence of our general formulas and of the computations for Artin groups contained in [D-S-S]. Finally in section 5 we collect some computations for cases $A_{2}$ and $A_{3}$, i.e., the symmetric groups $S_{3}$ and $S_{4}$, which we have performed using our complex. We omit the details of the computations.

\section{Definitions and main constructions}

1.1. Let $M$ be a real vector space of dimension $d+1$. We assume a basis $b_{1}, \ldots, b_{d+1}$ for $M$ has been fixed. In the sequel we shall denote by $M_{k}$ the subspace of $M$ having as basis the vectors $b_{1}, \ldots, b_{k}$, so that $M=M_{d+1}$. Let $N \in \mathbb{N}$ : by using the isomorphism $M^{N} \cong \mathbb{R}^{N} \otimes M$, each $P \in M^{N}$ is written as

$$
P=X_{1} \otimes b_{1}+\cdots+X_{d+1} \otimes b_{d+1}, \quad X_{k} \in \mathbb{R}^{N}, k=1, \ldots, d+1 .
$$

We will formally write $P \equiv X=X_{1}+\cdots+X_{d+1}$.

1.2. Let now $A=\left\{H_{j}\right\}_{j \in J}$ be a finite arrangement of linear hyperplanes in $\mathbb{R}^{N}, H_{j}=\left\{x \in \mathbb{R}^{N}: a_{j} . x=0\right\}, a_{j} \in \mathbb{R}^{N} \backslash\{0\}$. The $(d+1)$-complexification (or $M$-complexification) of $H_{j}$ is the linear subspace of $M^{N}$ :

$$
\left(H_{j}\right)_{M}:=H_{j} \otimes M=\left\{X: a_{j} \cdot X_{k}=0, k=1, \ldots, d+1\right\},
$$

so $\operatorname{codim}_{\mathbb{R}}\left(H_{j}\right)_{M}=d+1$ (this reduces to the standard complexification for $d=1)$. Set

$$
A_{M}:=\left\{\left(H_{j}\right)_{M}\right\}_{j \in J}
$$

and

$$
\mathbf{Y}^{(d+1)}:=\mathbf{Y}^{(d+1)}(A):=M^{N} \backslash \bigcup_{H \in A} H_{M}
$$


1.3. Let $\Phi:=\{F\}$ be the stratification of $\mathbb{R}^{N}$ induced by $A$ into facets ([Bou $]$ ). Then $\Phi$ is a poset with the partial ordering: $F \prec F^{\prime}$ iff $F^{\prime} \subset \operatorname{cl}(F)$. The atoms (called chambers) of $\Phi$ are the connected components of $\mathbb{R}^{N} \backslash \bigcup_{H \in A} H$. There exists a unique maximum for $\Phi$, namely the facet $L_{0}:=\bigcap_{H \in A} H$. It is convenient to consider also a rank function on $\Phi$, given by $\operatorname{rk}(F):=\operatorname{codim}(F)$. So a chamber is an element of rank 0 .

1.4. For every subset $L$ of $\mathbb{R}^{N}$, consider the arrangement $A_{L}=\{H \in A: L \subset H\}$. Let $\Phi_{L}$ be the induced stratification of $R^{N}$ (so $\left.\Phi=\Phi_{(\{0\})}\right)$. Fixing a facet $F$, let also $\Phi^{F}=\left\{F^{\prime} \in \Phi: F^{\prime} \prec F\right\}$. There is a map $p r_{F}: \Phi \rightarrow \Phi_{F}$ taking $F^{\prime}$ into the unique stratum containing it. Note that the restriction $\varphi_{F}:=p r_{F} \mid \Phi^{F}: \Phi^{F} \rightarrow \Phi_{F}$ is a dimension-preserving bijection.

Let then (for every $k \geq 0$ ) $\Phi^{k}$ be the product of $k$ copies of $\Phi$, and let

$$
\Phi^{(k)}=\left\{\left(F_{1}, \ldots, F_{k}\right) \in \Phi^{k}: F_{1} \succ \ldots \succ F_{k}\right\} .
$$

To each element $F=\left(F_{1}, \ldots, F_{d+1}\right)$, in $\Phi^{(d+1)}$ we associate the subset $\hat{F}$ of $M^{N}$ defined by

$$
\hat{F}:=\left\{X: X_{1} \in F_{1}, X_{k} \in \varphi_{F_{k-1}}\left(F_{k}\right), k=2, \ldots, d+1\right\} .
$$

Using arguments completely analogous to those of case $d=1$ (see [S0], [B-Z]) it is not difficult to show

\section{Proposition 1.4.1.}

(i) Each $\hat{F}$ is homeomorphic to an open cell.

(ii) $\bigcup_{F \in \Phi^{(d+1)}} \hat{F}=M^{N}$.

(iii) $\hat{F} \cap \hat{G}=\emptyset$ if $F \neq G$.

(iv) $\operatorname{cl}(\hat{F}) \cap \hat{G} \neq \emptyset$ iff $\operatorname{cl}(\hat{F}) \supset \hat{G}$.

(v) $\mathbf{Y}^{(d+1)}=\bigcup_{\left\{F: F_{d+1} \text { is a chamber of } \Phi\right\}} \hat{F}$.

Remark 1.4.2. Notice that Proposition 1.4 .1 easily implies that the collection $\left\{\hat{F}: F \in \Phi^{(d+1)}\right\}$ is a stratification of $M^{N}$. This stratification of $M^{N}$ is induced by the subspace arrangement of the $(d+1)$-complexified hyperplanes

$$
A_{M}:=\left\{H_{M}=H \otimes M: H \in A\right\} .
$$

(It is different from other stratifications which could be considered, like the product stratification).

In view of the above proposition, $\Phi^{(d+1)}$ becomes a poset with partial ordering $F \prec G \quad$ iff $\quad c l(\hat{F}) \supset \hat{G}$.

Lemma 1.4.3. For $F=\left(F_{1}, \ldots, F_{d+1}\right), G=\left(G_{1}, \ldots, G_{d+1}\right) \in \Phi^{(k)}$ one has $F \prec G$ iff $F_{1} \prec G_{1}$ and $p r_{F_{i}}\left(F_{i+1}\right) \prec p r_{F_{i}}\left(G_{i+1}\right)$ in the stratification $\Phi_{F_{i}}$, $i=1, \ldots, d$.

Proof. We have that $F \prec G$ iff $F_{1} \prec G_{1}$ and $\operatorname{cl}\left(\operatorname{pr}_{F_{i}}\left(F_{i+1}\right)\right) \supset \operatorname{pr}_{G_{i}}\left(G_{i+1}\right)$, $i=1, \ldots, d$. The left hand side is a union of strata in $\Phi_{F_{i}}$, one of which contains $G_{i+1}$, but $\operatorname{pr}_{F_{i}}\left(G_{i+1}\right)$ is by definition the stratum containing $G_{i+1}$, so one 
implication follows. Conversely one has $F_{1} \prec G_{1}$ and we have already seen in Proposition 1.4.1 (iv) that for $i=1, \ldots d, p_{G_{i}}\left(G_{i+1}\right) \subset p r_{F_{i}}\left(G_{i+1}\right)$. From this our claim is clear.

According to part (v) of proposition 1.4.1, the poset corresponding to the induced stratification of $\mathbf{Y}^{(d+1)}$ is

$$
\Phi_{0}^{(d+1)}:=\left\{F=\left(F_{1}, \ldots, F_{d+1}\right) \in \Phi^{(d+1)}: \operatorname{rk}\left(F_{d+1}\right)=0\right\} .
$$

The remaining elements of $\Phi^{(d+1)}$ are given by the poset

$$
\Phi_{+}^{(d+1)}:=\left\{F=\left(F_{1}, \ldots, F_{d+1}\right) \in \Phi^{(d+1)}: \operatorname{rk}\left(F_{d+1}\right)>0\right\}
$$

and correspond to a stratification of the union $\bigcup_{H \in A} H_{M}$ of the complexified subspaces.

Now choose one point $v(F)$ inside each stratum $\hat{F}$.

Proposition 1.4.4. For each chain $\mathcal{C}=\left(F^{0} \supsetneqq \cdots \supsetneqq F^{r}\right)$ of $\Phi^{(k)}$, the join in $\left(M_{k}\right)^{N}$

$$
s(\mathcal{C}):=\bigvee_{G \in \mathcal{C}} v(G)
$$

is an r-dimensional affine simplex. The set

$$
e(F):=\bigcup_{\mathcal{C} \prec F} s(\mathcal{C})
$$

where the union is over all chains $\mathcal{C}$ of $\Phi^{(k)}$ which have $F$ as an upper bound, is a triangulated cell in $\left(M_{k}\right)^{N}$ of dimension equal to $\operatorname{codim}(F)$. The cell $e(F)$ is dual to $F$, intersecting it in exactly one point. Its boundary is given by

$$
\partial(e(F))=\bigcup_{\mathcal{C} \prec F, F \notin \mathcal{C}} s(\mathcal{C})=\bigcup_{F^{\prime} \supsetneqq F} e\left(F^{\prime}\right) .
$$

The set $\mathbf{Q}^{(k)}:=\bigcup_{F \in \Phi^{(k)}} e(F)$, is a cellular $N^{\prime} k$-ball in $\left(M_{k}\right)^{N}$, linearly triangulated by (3), dual to the stratification. Here $N^{\prime}:=\operatorname{rk}\left(L_{0}\right)$ (see 1.2).

Proof. This follows as in [S0, part 1].

Remark 1.4.5. It follows from lemma 1.4.3 that if the last element $F_{d+1}$ of $F$ is a chamber, then also the last element of any $G \prec F$ is a chamber.

Remark 1.4.6. Let $F=\left(F_{1}, \ldots, F_{d+1}\right)$ and let $F_{j}$ be the first factor of $F$ which is equal to $F_{d+1}$. We can choose, using the basis of $1.1, v(F)=v\left(F_{1}\right) b_{1}+\cdots+v\left(F_{j}\right) b_{j}$ where $v\left(F_{i}\right) \in F_{i}$ is a point as in 1.3. Thus the choice of the $v\left(F_{i}\right)$ 's completely determines the realization of $\mathbf{Q}^{(d+1)}$. 
Definition. We denote by $\mathbf{X}^{(d)}$ the union of all cells in $\mathbf{Q}^{(d+1)}$ which correspond to $\Phi_{0}^{(d+1)}$.

It follows from remark 1.4.5 that $\mathbf{X}^{(d)}$ is a subcomplex of $\mathbf{Q}^{(d+1)}$ (actually, it is a regular cell complex in $M^{N}$ ).

Set $\Phi_{0} \subset \Phi$ as the set of chambers of $\Phi$. There is an inclusion $\Phi_{0}^{(d+1)} \subset$ $\Phi^{(d)} \times \Phi_{0}$ : indeed

$$
\Phi_{0}^{(d+1)}=\left\{F=\left(F^{\prime}, F_{d+1}\right) \in \Phi^{(d)} \times \Phi_{0}: F^{\prime}=\left(F_{1}, \ldots, F_{d}\right), F_{d} \succ F_{d+1}\right\}
$$

The map $\left(F^{\prime}, C\right) \rightarrow\left(F^{\prime}, \varphi_{F_{d-1}}^{-1} p r_{F_{d-1}}(C)\right)$ gives a "projection" $\Phi^{(d)} \times \Phi_{0} \rightarrow \Phi_{0}^{(d+1)}$ : we denote by $\left[F^{\prime}, C\right]$ the image of $\left(F^{\prime}, C\right)$.

Let us denote by $w(C)$ the point chosen in a chamber $C$. By using the previous projection, we can write a cell $e(F)$ of $\mathbf{X}^{(d)}, F=\left[F^{\prime}, C\right]$ as $e\left[F^{\prime}, C\right]$, (or $e[F, w]$, $w=w(C))$.

The "real projection"

$$
p r_{\Re}: M^{N} \rightarrow\left(M_{d}\right)^{N}: X_{1}+\cdots+X_{d+1} \rightarrow X_{1}+\cdots+X_{d}
$$

induces a map

$$
p r_{\Re}: \Phi^{(d+1)} \rightarrow \Phi^{(d)}: F=\left(F^{\prime}, F_{d+1}\right) \rightarrow F^{\prime}
$$

and a map

$$
p r_{\Re}: \mathbf{Q}^{(d+1)} \rightarrow \mathbf{Q}^{(d)}: e(F) \rightarrow e\left(F^{\prime}\right) .
$$

which restricts to a surjective map (which we continue to call $p r_{\Re}$ )

$$
p r_{\Re}: \mathbf{X}^{(d)} \rightarrow \mathbf{Q}^{(d)}: e\left[F^{\prime}, w\right] \rightarrow e\left(F^{\prime}\right) .
$$

In analogy with [S0], we have

\section{Theorem 1.4.7.}

(i) $\mathbf{X}^{(d)}$ is a deformation retract of $\mathbf{Y}^{(d+1)}$.

(ii) The map $\operatorname{pr}_{\Re} \mid e\left[F^{\prime}, w\right]$ is a homeomorphism between $e\left[F^{\prime}, w\right] \subset \mathbf{X}^{(d)}$ and $e\left(F^{\prime}\right) \subset \mathbf{Q}^{(d)}$. There are as many cells of $\mathbf{X}^{(d)}$ over $e\left(F^{\prime}\right)$ as the chambers $C \prec F_{d}$ (here $F^{\prime}=\left(F_{1}, \ldots, F_{d}\right)$ ).

(iii) $\partial\left(e\left[F^{\prime}, w\right]\right)=\bigcup \underset{\operatorname{codim}\left(F^{\prime \prime}\right)^{F^{\prime \prime}} \prec F^{\prime}}{\operatorname{codim}\left(F^{\prime}\right)-1} \quad e\left[F^{\prime \prime}, w\right]$.

(iv) Fixing $C \in \Phi_{0}$ the map $\psi_{w}: \mathbf{Q}^{(d)} \rightarrow \mathbf{X}^{(d)}(w=w(C))$ defined by $\left.\psi_{w}\right|_{e\left(F^{\prime}\right)}=\left(\left.p r_{\Re}\right|_{e\left[F^{\prime}, w\right]}\right)^{-1}$, is a cellular embedding of $\mathbf{Q}^{(d)}$ inside $\mathbf{X}^{(d)}$.

(v) $\operatorname{dim} e\left[F^{\prime}, w\right]=\operatorname{dim} e\left(F^{\prime}\right)=\operatorname{codim}_{\left(M_{d}\right)^{N}}\left(F^{\prime}\right)=\sum_{i=1}^{d} \operatorname{codim}\left(F_{i}\right)$. In particular, $\operatorname{dim}\left(\mathbf{X}^{(d)}\right)=\operatorname{dim}\left(\mathbf{Q}^{(d)}\right)=N d$. 
Proof. Part (i) is proved as in [S0] by an explicit contraction, or as in [B-Z].

As a preliminary step remark that decomposing $M^{N}=S_{0} \oplus S_{0}^{\perp}$ as orthogonal direct sum, where $S_{0}$ is the stratum associated to the unique maximum $\left(L_{0}, \ldots, L_{0}\right)$ of $\Phi^{(d+1)}$, one reduces to the case $L_{0}=\{\mathbf{0}\}$ (where $\mathbf{0}$ is the origin in $\mathbb{R}^{N}$ ). In fact, one can choose $\mathbf{Q}^{(d+1)} \subset S_{0}^{\perp}$, and the obvious linear contraction of $M^{N}$ into $S_{0}^{\perp}$ preserves all the strata. So assume from now on that $L_{0}=\{\mathbf{0}\}$.

Now notice that the PL-ball $\mathbf{Q}^{(d+1)}$ is star-shaped with respect to the origin of $M^{N}$ since for each simplex, by our assumption and Proposition 1.4.4, $s(\mathcal{C})$ of $\mathbf{Q}^{(d+1)},\{0\} \vee s(\mathcal{C})$ is also a simplex of $\mathbf{Q}^{(d+1)}$. This implies, similarly to Lemma 1.3.1, that $\mathbf{Q}^{(d+1)}$ is a deformation retract of $M^{N}$ via "radial" contraction.

It is clear that such a contraction preserves all strata, and in particular $\mathbf{Y}^{(d+1)}$ contracts onto $\mathbf{Q}^{(d+1)} \cap \mathbf{Y}^{(d+1)}$ and $Z:=\bigcup_{H \in A} H_{M}$ contracts onto $\mathbf{Q}^{(d+1)} \cap Z$.

By Proposition 1.4.4, $\mathbf{Q}^{(d+1)}$ is a simplicial complex with vertices $v(F)$, $F \in \Phi^{(d+1)}$. One has that $\mathbf{X}^{(d)}$ is the subcomplex generated by the vertices $v(F), F \in \Phi_{0}^{(d+1)}$, while the remaining vertices $v(F), F \in \Phi_{+}^{(d+1)}$, generate a subcomplex $K$ such that $|K|=\mathbf{Q}^{(d+1)} \cap Z$. Fix a simplex $\sigma$ in $\mathbf{Q}^{(d+1)}$, set $\sigma_{0}$ (resp. $\left.\sigma_{1}\right)$ equal to the face of $\sigma$ generated by the vertices in $\Phi_{0}^{(d+1)}\left(\operatorname{resp} . \Phi_{+}^{(d+1)}\right.$ ) $\left(\sigma_{1}\right.$ can be empty). Then $\sigma-\sigma_{1}$ has a canonical contraction onto $\sigma_{0}$ defined as follows: for each $p \in \sigma-\sigma_{1}$ the affine space generated by $p$ and $\sigma_{1}$ meets $\sigma_{0}$ in a single point $p_{0}$ and we set, for $t \in[0,1], H_{\sigma}(p, t)=t p_{0}+(1-t) p$. Patching together the various $H_{\sigma}$ we deduce that $\mathbf{X}^{(d)}$ is deformation retract of

$$
\mathbf{Q}^{(d+1)} \backslash|K|=\mathbf{Q}^{(d+1)} \cap \mathbf{Y}^{(d+1)} .
$$

(ii) The real projection induces a codimension preserving map between $\Phi_{0}^{(d+1)}$ and $\Phi^{(d)}$. One can realize an explicit homeomorphism between $e\left[F^{\prime}, w\right]$ and $e\left(F^{\prime}\right)$ by an affine homotopy taking the point $v(G)$ into the point $v\left(p r_{\Re}(G)\right)$. The second assertion in (ii) is trivial.

(iii) From lemma 1.4.3, setting $w=w(C)$ and $w^{\prime}=w^{\prime}\left(C^{\prime}\right),\left[F^{\prime \prime}, w^{\prime}\right] \prec\left[F^{\prime}, w\right]$ iff $F^{\prime \prime} \prec F^{\prime}$ and $p r_{F_{d}^{\prime \prime}}\left(C^{\prime}\right) \prec p r_{F_{d}^{\prime \prime}}(C)$. If this holds then $\left[F^{\prime \prime}, w^{\prime}\right]=\left[F^{\prime \prime}, w\right]$.

(iv) It is sufficient to observe that $\psi_{w}$ is well defined: if $G^{\prime} \prec F^{\prime}$ then $\left.\left(\left.\psi_{w}\right|_{e\left(F^{\prime}\right)}\right)\right|_{e\left(G^{\prime}\right)}=\psi_{w} \mid e\left(G^{\prime}\right)$ by (iii).

$(\mathrm{v})$ is clear.

Remark 1.4.8. All results of this paper generalize to the case where $A$ is locally finite arrangement of affine hyperplanes in $\mathbb{R}^{N}$ (or in subcone in $\mathbb{R}^{N}$ ). It is possible to find a contraction $\mathbf{Y}^{(d+1)} \rightarrow \mathbf{X}^{(d)}$ which generalizes that constructed in $[\mathrm{S} 0]$.

1.5. We consider now the natural inclusion $j_{d}:\left(M_{d}\right)^{N} \rightarrow\left(M_{d+1}\right)^{N}$. It induces an injection of posets $j_{d}: \Phi^{(d+1)} \rightarrow \Phi^{(d+2)}$ :

$$
F=\left(F_{1}, \ldots, F_{d+1}\right) \rightarrow F^{1}=\left(F_{1}, \ldots, F_{d+1}, F_{d+1}\right),
$$


so $\hat{F}^{1}$ is the unique stratum of $\Phi^{(d+2)}$ which contains $\hat{F}$. This map preserves the codimension iff $F_{d+1} \in \Phi_{0}$, i.e., iff $F \in \Phi_{0}^{(d+1)}$. The induced map on the dual (call it again $j_{d}$ )

$$
j_{d}: \mathbf{Q}^{(d+1)} \rightarrow \mathbf{Q}^{(d+2)}: e\left(F_{1}, \ldots F_{d+1}\right) \rightarrow e\left(F_{1}, \ldots, F_{d+1}, F_{d+1}\right),
$$

is a dimension preserving cellular map when restricted to $\mathbf{X}^{(d)}$, so it identifies $\mathbf{X}^{(d)}$ to a subcomplex of $\mathbf{X}^{(d+1)}$ (see (2), (3) and remark 1.4.6 of part 1.4).

Theorem 1.5.1. $\mathbf{X}^{(d+1)}$ is obtained from $\mathbf{X}^{(d)}$ by attaching cells of dimension grater or equal than $d+1$.

Proof. A cell $e\left(F_{1}, \ldots, F_{d+2}\right) \in \mathbf{X}^{(d+1)}$ belongs to $\mathbf{X}^{(d+1)} \backslash \mathbf{X}^{(d)}$ iff $F_{d+1} \notin \Phi_{0}$. Then also $F_{i} \notin \Phi_{0}$ for $1 \leq i \leq d+1$, therefore, $\operatorname{dim} e(F)=\sum_{i=1}^{d+2} \operatorname{codim} F_{i} \geq d+1$.

Notice that there are cells of dimension $d+1$ in $\mathbf{X}^{(d+1)} \backslash \mathbf{X}^{(d)}$, precisely of the form $e(F, \ldots, F, C), \operatorname{codim}(F)=1, C \in \Phi_{0}$. Of course the space $\mathbf{X}^{(\infty)}: \lim _{d \rightarrow \infty} \mathbf{X}^{(d)}$, is contractible since $\mathbf{Y}^{(d+1)}$ is obtained from $M^{N}$ by removing subspaces of codimension $d+1$. So by theorem 1.5.1 we get

Corollary 1.5.2. The space $\mathbf{X}^{(d)}$ is $d-1$ connected.

\section{The case of reflection arrangements}

2.1. Let now $\mathbf{W}$ be a group generated by reflections in $\mathbb{R}^{N}$. For notational simplicity, we assume $\mathbf{W}$ is finite and acts essentially on $\mathbb{R}^{N}$ (for general Coxeter groups, one should restrict the action of the group to a cone, e.g., the Tits cone). Let $A$ be the reflection arrangement associated to $\mathbf{W}$, that is

$$
A=\{H: H \text { is the fixed point set of some reflection in } W\} \text {. }
$$

W acts on $\left(M_{k}\right)^{N}$ diagonally:

$$
w \cdot\left(X_{1}+\cdots+X_{k}\right)=w \cdot X_{1}+\cdots+w \cdot X_{k}, \quad k=1, \ldots, d+1 .
$$

We can build $\mathbf{Q}=\mathbf{Q}^{(1)}$ invariant for the action of $\mathbf{W}$ as follows: choose a chamber $C_{0}$ and let $w_{0} \in C_{0}$. Let $\mathbf{Q}$ be the convex hull of the $\mathbf{W}$-orbit of $w_{0}$. Then $\mathbf{Q}$ has a natural structure of cell complex, with 0 - cells corresponding to the orbit of $w_{0}$ : each point $v(F), F \in \Phi$ (see 1.3), is determined as the orthogonal projection into $F$ of any $w=w(C), C \in \Phi_{0}$, with $F \prec C$. By choosing the points $v(F)$ as in remark 1.4.6, $w_{0}$ determines also the cellular structure of $\mathbf{Q}^{(k)}, k=1, \ldots, d+1$.

\section{Theorem 2.1.1.}

(i) The stabilizer $\mathbf{W}_{X}$ of a point $X \equiv X_{1}+\cdots+X_{k} \in\left(M_{k}\right)^{N}$ is the subgroup generated by the reflections corresponding to $A_{X}:=A_{\left\{X_{1}\right\}} \cap \cdots \cap A_{\left\{X_{k}\right\}}$. 
The stabilizer $\mathbf{W}_{F}$ of $\hat{F}, F=\left(F_{1}, \ldots, F_{k}\right) \in \Phi^{(k)}$, is the stabilizer of any $X \in \hat{F}$, and it is the subgroup generated by the reflections corresponding to $A_{F_{k}}$.

(ii) $\mathbf{W}$ acts on the stratification $\Phi^{(k)}$ by $\gamma \cdot \hat{F}=\hat{G}, F=\left(F_{1}, \ldots, F_{k}\right), G=$ $\left(\gamma \cdot F_{1}, \ldots, \gamma \cdot F_{k}\right)$ and on $\mathbf{Q}^{(k)}$ by $\gamma \cdot e(F)=e(\gamma \cdot F)(k=1, \ldots, d+1)$. The $\mathbf{W}$-action is free on $\mathbf{Y}^{(d+1)}$ and on $\mathbf{X}^{(d)}$.

(iii) The orbit space $\mathbf{X}_{\mathbf{W}}^{(d)}:=\mathbf{X}^{(d)} / \mathbf{W}$ is a deformation retract of the orbit space $\mathbf{Y}^{(d+1)} / \mathbf{W}$.

Proof. (i) The stabilizer of any subset $S \subset \mathbb{R}^{N}$ is the subgroup generated by the reflections with respect to the hyperplanes of $A$ which contain $S$ ([Bou]). Since the stabilizer of $X \equiv X_{1}+\cdots+X_{k}$ is the stabilizer of $\left\{X_{1}, \ldots, X_{k}\right\} \subset \mathbb{R}^{N}$, the first part of (i) follows. The second follows because the hyperplanes in $A_{F_{k}}$ are precisely those which contain all $F_{i}, 1 \leq i \leq k$.

(ii) The $\mathbf{W}$-action on $\Phi^{(k)}$ is clear. By the choice of the points $v \in \hat{G}, \Gamma \in \Phi^{(k)}$, a given $\gamma \in \mathbf{W}$ maps the cell $e(F)$ to the cell $e(\gamma \cdot F)$.

The $\mathbf{W}$-action on $\mathbf{Y}^{(d+1)}$ is free by (i) (for all strata in $\mathbf{Y}^{(d+1)}$, the last factor is a chamber). Similarly for $\mathbf{X}^{(d)}$.

(iii) This comes immediately from part (i) of theorem 1.4.7, since the retractions which are defined there are clearly equivariant.

2.2. We want to give a new combinatorial description of the stratification $\Phi^{(d)}$ which will make the action of $\mathbf{W}$ more explicit. We need some more notations and results.

Let $S$ be the set of reflections with respect to the walls of the base chamber $C_{0}$ : then the pair $(\mathbf{W}, S)$ is a Coxeter system (see [Bou]) and each stratum $F$ of $\Phi$ corresponds to a unique left-coset of a parabolic subgroup $\mathbf{W}_{\Gamma}$, where $\Gamma \subset S$ is the type of $F$. The action of $\mathbf{W}$ on $\Phi$ corresponds to the natural action of $\mathbf{W}$ on the cosets. Recall also ([Hum]) that each coset contains exactly one element of minimal length (the length being measured with respect to the system $S$ of generators): let $\mathbf{W}^{\Gamma}$ be the set of minimal coset representatives for the cosets of $\mathbf{W}_{\Gamma}$. Then each coset is uniquely determined by its type $\Gamma \subset S$ and by an element $\gamma \in \mathbf{W}^{\Gamma}$. Let $H_{s} \in A$ be the wall of $C_{0}$ which is fixed by $s \in S$; let $H_{\Gamma}:=\cap_{s \in \Gamma} H_{s}$ and let $F_{\Gamma}$ be the facet (= stratum) of $c l\left(C_{0}\right)$ of type $\Gamma$, i.e., with linear span $H_{\Gamma}$. The relation between a stratum $F \in \Phi$ and its corresponding pair $(\gamma, \Gamma)$ is given by $F=\gamma \cdot F_{\Gamma}$.

More generally one denotes by $\mathbf{W}_{\Gamma^{\prime}}^{\Gamma}, \Gamma \subset \Gamma^{\prime}$, the set of minimal coset representatives of $\mathbf{W}_{\Gamma}$ in $\mathbf{W}_{\Gamma^{\prime}}$ (with respect to the set of generators $\Gamma^{\prime} \subset S$; so $\left.\mathbf{W}^{\Gamma}=\mathbf{W}_{S}^{\Gamma}\right)$.

Proposition 2.2.1. Let $F^{\prime}, F \in \Phi$ correspond to the pairs $(\gamma, \Gamma),\left(\gamma^{\prime}, \Gamma^{\prime}\right)$ respectively. Then if $F^{\prime} \succ F F^{\prime}=\gamma \cdot F_{\Gamma^{\prime}}, F=\gamma \cdot F_{\Gamma}$. In particular $\Gamma^{\prime}$ and $\gamma$ determine $F^{\prime}$.

Moreover, the following conditions hold:

a) $\Gamma^{\prime} \supset \Gamma$, 
b) $\gamma=\gamma^{\prime} \gamma^{\prime \prime}, \gamma^{\prime \prime} \in \mathbf{W}_{\Gamma^{\prime}}^{\Gamma}$.

Conversely, if a) and b) hold, then $F^{\prime} \succ F$.

Proof. By definition of $\gamma, \Gamma$ one has $F=\gamma . F_{\Gamma}$. Since $F^{\prime} \succ F$, then $\gamma^{-1}\left(F^{\prime}\right) \succ \gamma^{-1}(F)=F_{\Gamma}$ so $\gamma^{-1}\left(F^{\prime}\right)=F_{\Gamma^{\prime}}$ since the $\gamma^{-1}\left(F^{\prime}\right)$ belongs to the closure of the base chamber $C_{0}$. This also implies $\Gamma^{\prime} \supset \Gamma$. Since $\left(\gamma^{\prime}\right)^{-1}\left(F^{\prime}\right)=F_{\Gamma^{\prime}}$ and (see $\left.[\mathrm{Hum}]\right) \mathbf{W}^{\Gamma}=\mathbf{W}^{\Gamma^{\prime}} \mathbf{W}_{\Gamma^{\prime}}^{\Gamma}$, the last formula of our proposition also follows.

Conversely, $F_{\Gamma^{\prime}} \succ F_{\Gamma}, \gamma^{\prime \prime} \in \mathbf{W}_{\Gamma^{\prime}}^{\Gamma}$ implies $\left(\gamma^{\prime}\right)^{-1} \cdot F^{\prime}=F_{\Gamma^{\prime}} \succ \gamma^{\prime \prime} F_{\Gamma}=\left(\gamma^{\prime}\right)^{-1} \cdot F$.

Notice that is easy to see that condition b) of the previous proposition is equivalent to: $\left(\gamma^{\prime}\right)^{-1} \gamma \in \mathbf{W}_{\Gamma^{\prime}}$.

From the previous proposition we immediately deduce

Corollary 2.2.2. A stratum $F \in \Phi^{(d)}$ is uniquely determined by a pair $(\gamma, \Gamma)$ where $\Gamma=\left(\Gamma_{1} \supset \cdots \supset \Gamma_{d}\right), \Gamma_{i} \subset S$, is a flag of subsets in $S$ (or a chain in the poset $S$, endowed with the natural ordering) and $\gamma \in \mathbf{W}^{\Gamma_{d}}$.

By the above discussion, it follows that the set $\Phi^{(d)}$ is in bijection with the set $\left\{(\gamma, \Gamma): \Gamma=\left(\Gamma_{1} \supset \ldots \supset \Gamma_{d}\right), \gamma \in \mathbf{W}^{\Gamma_{d}}\right\}$. From now on, if $F \in \Phi^{(d)}$ corresponds to the pair $(\gamma, \Gamma)$, we shall denote the cell $e(F) \in \mathbf{Q}^{(d)}$ also by $e(\gamma, \Gamma)$.

We now have

Proposition 2.2.3. Two cells $e(\gamma, \Gamma)$ and $e\left(\gamma^{\prime}, \Gamma^{\prime}\right)$ of $\mathbf{Q}^{(d)}$ belong to the same orbit iff $\Gamma=\Gamma^{\prime}$.

Proof. The action of $\mathbf{W}$ preserves the type, so our condition is necessary. Conversely, if $e(F) \leftrightarrow(\gamma, \Gamma)$ then $\gamma^{-1} . e(F)$ is the unique cell equivalent to $e(F)$ whose dual stratum "belongs" to $\operatorname{cl}\left(C_{0}\right)$. From this our claim follows.

Definition. Given $F=\left(F_{1}, \ldots, F_{d}\right) \in \Phi^{(d)}$, set $C_{0}(F):=\left\{C \in \Phi_{0}: C\right.$ and $C_{0}$ lye in the same chamber of the arrangement $A_{F_{d}}$.

By using our identification of $\Phi_{0}$ with $\mathbf{W}$, we can see $C_{0}(F)$ as a set of elements of $\mathbf{W}$. Notice that $A_{F_{d}}=\emptyset \Leftrightarrow F_{d}$ is a chamber $\Leftrightarrow C_{0}(F)=\Phi_{0}(\leftrightarrow \mathbf{W})$. It is convenient to express also the set $C_{0}(F), F \in \Phi^{(d)}$, in more intrinsic terms.

If $\Gamma \subset S$, let us consider the set $\{w \in \mathbf{W}: l(s w)>l(w)$, for all $s \in \Gamma\}$ of minimal coset representatives for the right-cosets $\mathbf{W}_{\Gamma} . w$. It is clear that such a set coincides with the set $\left(\mathbf{W}^{\Gamma}\right)^{-1}$ of the inverses of the elements in $\mathbf{W}^{\Gamma}$.

Lemma 2.2.4. Let $F=\left(F_{1}, \ldots, F_{d}\right) \in \Phi^{(d)}$ correspond to the pair $(\gamma, \Gamma)$, $\gamma \in \mathbf{W}^{\Gamma_{d}}$. Then

$$
C_{0}(F)=\gamma \cdot\left(\mathbf{W}^{\Gamma_{d}}\right)^{-1}
$$

Proof. If $F_{d}$ is in the closure of $C_{0}$ (iff $\gamma=1$ ) then one verifies easily that the condition $l(s w)>l(w)$, for all $s \in \Gamma_{d}$, is equivalent to $w \in C_{0}(F)$ (see also $[\mathrm{Bou}])$. So (5) is true in this case. 
In the general case, notice that $\gamma^{-1} . F$ is in the closure of $C_{0}$ and

$$
C_{0}(F)=\gamma \cdot C_{0}\left(\gamma^{-1} \cdot F\right)=\gamma \cdot\left(\mathbf{W}^{\Gamma_{d}}\right)^{-1} .
$$

This is our claim.

2.3. If we apply the above considerations to the cells of $\mathbf{X}^{(d)}$, we have that such a cell is of the form $e(\gamma, \Gamma \supset \emptyset)$ with $\Gamma=\left(\Gamma_{1} \supset \ldots \supset \Gamma_{d}\right)$ and $\gamma \in \mathbf{W}^{\emptyset}=\mathbf{W}$. The action of $\mathbf{W}$ is written as

$$
w \cdot e(\gamma, \Gamma \supset \emptyset)=e(w \gamma, \Gamma \supset \emptyset),
$$

where the factor $w \cdot \gamma$ in (5) is just multiplication in $\mathbf{W}$.

Since $\mathbf{W}$ acts essentially, the stratum $\{\mathbf{0}\}$ ( 0 being the origin of $\left.\left(M_{d}\right)^{N}\right)$ is the unique maximum in $\Phi^{(d)}$. It corresponds to the sequence $S^{d}:=(\underbrace{S \supset S \cdots \supset S}_{d-\text { times }})$.

Lemma 2.3.1. The $N d$-cell $E_{0}:=e\left(1, S^{d} \supset \emptyset\right)$ (here $1 \in \mathbf{W}$ is the unit) intersects all $\mathbf{W}$-orbits.

Proof. It follows from theorem 1.4.7, (iii), that the boundary of $E_{0}$ contains all cells of the form $e(1, \Gamma \supset \emptyset)$. This gives our claim.

Using theorem 1.4.7, (iv), consider the map $\psi_{w_{0}}: \mathbf{Q}^{(d)} \rightarrow \mathbf{X}^{(d)}$ which identifies $\mathbf{Q}^{(d)}$ with $E_{0}$.

Proposition 2.3.2. Let $e(\gamma, \Gamma)$ be a cell of $\mathbf{Q}^{(d)}$. Then $\psi_{w_{0}}(e(\gamma, \Gamma))=e(\gamma, \Gamma \supset \emptyset)$. Proof. A cell $e(\gamma, \Gamma)$ of $\mathbf{Q}^{(d)}$ is taken homeomorphically onto a cell $e(\bar{\gamma}, \Gamma \supset \emptyset)$ of $\mathbf{X}^{(d)}$, with $\bar{\gamma}^{-1} \gamma$ lying in the subgroup $\mathbf{W}_{\Gamma_{d}}$. On the other hand, since $e(\bar{\gamma}, \Gamma \supset \emptyset)$ lies in $E_{0}$, if $F$ corresponds to $(\gamma, \Gamma), \bar{\gamma}^{-1} C_{0}(F)$ should contain the identity element. By Lemma 2.2.4 this means that $\gamma^{-1} \bar{\gamma} \in \mathbf{W}_{\Gamma_{d}} \cap \mathbf{W}^{\Gamma_{d}}=\{1\}$.

For notational simplicity, we shall identify a cell of $\mathbf{Q}^{(d)}$ with its $\psi_{w_{0}}$-image in $\mathbf{X}^{(d)}$.

Summarizing we have obtained the following description of the orbit space $\mathbf{X}_{\mathbf{W}}^{(d)}$.

Theorem 2.3.4. The orbit space $\mathbf{X}_{\mathbf{W}}^{(d)}$ can be described as the quotient of the $N d$-dimensional convex polyhedron $\mathbf{Q}^{(d)}$ under the equivalence relation which identifies two cells $e(\gamma, \Gamma), e\left(\gamma^{\prime}, \Gamma\right)$, using the homeomorphism of $\left(M_{d}\right)^{N}$ induced by the element $\gamma^{\prime} \gamma^{-1}$.

\section{Definition.}

1) We set $S^{(d)}:=\left\{\Gamma=\left(\Gamma_{1} \supset \cdots \supset \Gamma_{d}\right): \Gamma_{i} \subset S\right\}$, endowed with the natural partial ordering $\Gamma=\left(\Gamma_{1} \supset \cdots \supset \Gamma_{d}\right) \prec \Gamma^{\prime}=\left(\Gamma_{1}^{\prime} \supset \cdots \supset \Gamma_{d}^{\prime}\right)$ iff $\Gamma_{i} \subset \Gamma_{i}^{\prime}, \forall i$

Set also $S_{k}^{(d)}:=\left\{\Gamma \in S^{(d)}:|\Gamma|=k\right\}$ where $|\Gamma|:=\sum_{i \geq 1}\left|\Gamma_{i}\right|$.

2) Let $\pi_{\mathbf{W}}: \mathbf{Q}^{(d)} \rightarrow \mathbf{X}_{\mathbf{W}}^{(d)}$ be the projection onto the quotient. For any cell $e(\gamma, \Gamma)$ of $\mathbf{Q}^{(d)}$ we shall denote the cell $\pi_{\mathbf{W}}(e(\gamma, \Gamma)) \in \mathbf{X}_{\mathbf{W}}^{(d)}$ by $e(\Gamma)$. 
From preceding theorem and theorem 1.4 .7 we immediately get

\section{Corollary 2.3.5.}

(i) The cells of $\mathbf{X}_{\mathbf{W}}^{(d)}$ are in one to one correspondence with $S^{(d)}$.

(ii) $\operatorname{dim}(e(\gamma, \Gamma))=\operatorname{dim}(e(\Gamma))=\sum_{i=1}^{d}\left|\Gamma_{i}\right|$.

(iii) The $k$-cells of $\mathbf{X}_{\mathbf{W}}^{(d)}$ are in one to one correspondence with $S_{k}^{(d)}$, and their number $\nu_{k}$ is the number of solutions of

$$
a_{1}+\cdots+a_{N}=k, \quad 0 \leq a_{i} \leq d, i=1, \ldots, N .
$$

Therefore for $d \geq k$

$$
\nu_{k}=\left(\begin{array}{c}
N+k-1 \\
k
\end{array}\right) .
$$

For $d=1$ we re-find results in [S1]. Notice that there is just one 0-cell, corresponding to the class of $w_{0}$, and $\mathrm{N}$ 1-cells, corresponding to the classes of the 1-cells $e(F)$, where $F$ is a codimensional 1 facet of $C_{0}$. The 2-cells are (for $d \geq 2)$ of two types: the type $(\Gamma, \emptyset, \ldots, \emptyset)$, with $|\Gamma|=2$, which corresponds to the class of a 2-cells $e(F), F$ being a codimensional 2 facet of $C_{0}$ of type $\Gamma$; the type $(\Gamma, \Gamma, \emptyset, \ldots, \emptyset)$, with $|\Gamma|=1$.

The action of $W$ commutes with the inclusion of $\mathbf{X}^{(d)}$ into $\mathbf{X}^{(d+1)}$ defined in 1.5 , so $\mathbf{X}_{\mathbf{W}}^{(d)}$ embeds as a subcomplex of $\mathbf{X}_{\mathbf{W}}^{(d+1)}$. From theorem 1.5.1 it follows

Corollary 2.3.6. The orbit space $\mathbf{X}_{\mathbf{W}}^{(d+1)}$ is obtained from $\mathbf{X}_{\mathbf{W}}^{(d)}$ by attaching cells of dimension bigger or equal than $d+1$.

Notice that the inclusion of $\mathbf{X}_{\mathbf{W}}^{(d)}$ into $\mathbf{X}_{\mathbf{W}}^{(d+1)}$ is given by

$$
e\left(\Gamma_{1} \supset \cdots \supset \Gamma_{d}\right) \rightarrow e\left(\Gamma_{1} \supset \cdots \supset \Gamma_{d} \supset \emptyset\right) .
$$

\section{Computation of cohomology}

3.1. In order to compute the cohomology of the orbit space $\mathbf{X}_{\mathbf{W}}^{(d)}$ considered in the previous section, we have to compute the boundary of each cell: this is done by characterizing the cells in the boundary of a given cell, and by assigning an orientation to all cells. If $\Gamma$ is a d-chain of $S$, let us give an orientation to the cell $e(1, \Gamma)$ and then we give orientation to a cell $e(\gamma, \Gamma \supset \emptyset)$ by requiring that $\gamma$ is orientation preserving. Therefore one gets an orientation for the cells of $\mathbf{X}_{\mathbf{W}}^{(d)}$.

We need some technical lemma.

Lemma 3.1.1. Given $F=\left(F_{1}, \ldots, F_{d}\right), G=\left(G_{1}, \ldots, G_{d}\right) \in \Phi^{(d)}$ such that each $F_{i}, G_{j} \in \operatorname{cl}\left(C_{0}\right), \forall i, j$, that is iff $F_{d}, G_{d} \in \operatorname{cl}\left(C_{0}\right)$, one has $F \prec G$ iff $F_{i} \prec G_{i}$, in $\Phi, i=1, \ldots, d$.

Proof. This follows from lemma 1.4 .3 by observing that

$$
\operatorname{cl}\left(\operatorname{pr}_{F_{i-1}}\left(F_{i}\right)\right) \cap \operatorname{cl}\left(C_{0}\right)=\operatorname{cl}\left(F_{i}\right) .
$$

Notice that by the above Lemma, there is an order preserving bijection between the subset of $\Phi^{(d)}$ of the d-chains of facets of $C_{0}$ and $S^{(d)}$. 
Lemma 3.1.2. For $\Gamma, \Gamma^{\prime} \subset S$ and $\beta \in \mathbf{W}^{\Gamma^{\prime}}$ the following conditions are equivalent:

1) $\beta^{-1} \mathbf{W}_{\Gamma} \beta \subset \mathbf{W}_{\Gamma^{\prime}}$;

2) $\beta^{-1} \Gamma \beta \subset \Gamma^{\prime}$.

Proof. 2) $\Rightarrow 1$ ) is trivial. So, assume 1). Let $\ell: \mathbf{W} \rightarrow \mathbb{Z}_{\geq 0}$ denote the length function with respect to $S$. Let $s \in \Gamma$. Set $t=\beta^{-1} s \beta \in \mathbf{W}_{\Gamma^{\prime}}$. Since $\beta \in \mathbf{W}^{\Gamma^{\prime}}$ we have $\ell(\beta t)=\ell(\beta)+\ell(t)$. On the other hand, $\ell(\beta t)=\ell(s \beta) \leq \ell(s)+\ell(\beta)=$ $\ell(\beta)+1$. It follows that $\ell(t)=1$ that is $t \in S \cap \mathbf{W}_{\Gamma^{\prime}}=\Gamma^{\prime}$ as desired.

Theorem 3.1.3. Let $e=e(\gamma, \Gamma \supset \emptyset)$ be a cell in $\mathbf{X}^{(d)}$ of dimension $\lambda=|\Gamma|$. $A(\lambda-1)-$ cell $e^{\prime}=e\left(\gamma^{\prime}, \Gamma^{\prime} \supset \emptyset\right)$ is in the boundary of $e$, if, setting $\beta=\gamma^{-1} \gamma^{\prime}$, there is a unique $1 \leq i \leq d$ such that

A) If $k<i, \Gamma_{k}^{\prime}=\Gamma_{k}$;

B) $\Gamma_{i}^{\prime}=\Gamma_{i}-\{\tau\}$, for some $\tau \in \Gamma_{i}$, and $\beta^{-1} \Gamma_{i+1} \beta \subset \Gamma_{i}^{\prime}$.

C) If $k>i, \Gamma_{k}^{\prime}=\beta^{-1} \Gamma_{k} \beta$. Moreover, $\beta \in \mathbf{W}_{\Gamma_{i}}^{\Gamma_{i}^{\prime}}$.

Viceversa, given $\tau \in \Gamma_{i}$ and $\beta \in \mathbf{W}_{\Gamma_{i}}^{\Gamma_{i}-\{\tau\}}$, satisfying $B$ ) and $\left.C\right)$, there is a cell

$$
e^{\prime}=e\left(\gamma \beta, \Gamma_{1}, \ldots, \Gamma_{i-1}, \Gamma_{i}-\{\tau\}, \beta^{-1} \Gamma_{i+1} \beta, \ldots, \beta^{-1} \Gamma_{d} \beta, \emptyset\right) \subset \partial e
$$

Proof. By equivariance we can assume that $\gamma=1$, so that $e(1, \Gamma \supset \emptyset)$ lies in $E_{0}$ and, using $\psi_{w_{0}}$ it suffices to determine the boundary of $e=e(1, \Gamma)$ in $\mathbf{Q}^{(d)}$. Assume that $e^{\prime}:=e\left(\beta, \Gamma^{\prime}\right)$ is a $\lambda$-1-dimensional cell lying in the boundary of $e$. Let $e$ correspond to $F=\left(F_{1} \succ \ldots \succ F_{d}\right)$ and $e^{\prime}$ to $F^{\prime}=\left(F_{1}^{\prime} \succ \ldots \succ F_{d}^{\prime}\right)$. By lemma $1.4 .3 e^{\prime} \subset \partial e$ if and only if

$$
c l\left(p r_{F_{k-1}^{\prime}}\left(F_{k}^{\prime}\right)\right) \supset p r_{F_{k-1}^{\prime}}\left(F_{k}\right), \quad k=1, \ldots, d
$$

Since we have $\sum \operatorname{dim}\left(F_{k}^{\prime}\right)=1+\sum \operatorname{dim}\left(F_{k}\right)$ and

$$
\operatorname{dim}\left(F_{k}^{\prime}\right)=\operatorname{dim}\left(p r_{F_{k-1}^{\prime}}\left(F_{k}^{\prime}\right)\right) \geq \operatorname{dim}\left(p r_{F_{k-1}^{\prime}}\left(F_{k}\right)\right) \geq \operatorname{dim}\left(F_{k}\right),
$$

we deduce that there exists $i$ such that

$$
\operatorname{dim}\left(F_{i}^{\prime}\right)=\operatorname{dim}\left(F_{i}\right)+1, \quad \operatorname{dim}\left(F_{k}^{\prime}\right)=\operatorname{dim}\left(F_{k}\right), \quad k \neq i .
$$

From (7) we get

$$
p r_{F_{k-1}^{\prime}}\left(F_{k}^{\prime}\right)=p r_{F_{k-1}^{\prime}}\left(F_{k}\right), \quad k \neq i
$$

(we set to simplify notations $F_{0}=0$ ) and therefore an equality of supports

$$
\left|F_{k}^{\prime}\right|=\left|F_{k}\right|, \quad k \neq i
$$


Part A) then follows by induction on $k$ since $p r_{F_{k-1}^{\prime}}$ is a dimension preserving bijection when restricted to strata $G \prec F_{k-1}^{\prime}$.

From (7) applied when $k=i$ we deduce $F_{i}^{\prime} \prec F_{i}$. This condition implies (prop. 2.2.1) that $\Gamma_{i}^{\prime} \subset \Gamma_{i}$ and that $\beta=\gamma^{\prime} \in \mathbf{W}_{\Gamma_{i}} \cdot \operatorname{dim}\left(F_{i}^{\prime}\right)=\operatorname{dim}\left(F_{i}\right)+1$ clearly gives $\left|\Gamma_{i}^{\prime}\right|=\left|\Gamma_{i}\right|-1$. Since by (9) $\left|F_{i+1}^{\prime}\right|=\left|F_{i+1}\right|$, we get

$$
\left|F_{i+1}\right| \supset\left|F_{i}^{\prime}\right|
$$

and this translates into $\beta^{-1} \mathbf{W}_{\Gamma_{i+1}} \beta \subset \mathbf{W}_{\Gamma_{i}^{\prime}}$. By Lemma 3.1.2 this finishes the proof of B).

Suppose $k>i$. The equality (9) of supports clearly implies that $\beta^{-1} \mathbf{W}_{\Gamma_{k}} \beta=$ $\mathbf{W}_{\Gamma_{k}^{\prime}}$, and again by Lemma 3.1.2 we get the first claim in $\mathrm{C}$ ).

Recall that, by definition $\beta \in \mathbf{W}^{\Gamma_{d}^{\prime}}$. Assume now by reverse induction that $\beta \in \mathbf{W}^{\Gamma_{k}^{\prime}}$ for some $i+1 \leq k \leq d$. Now notice that since $p r_{F_{k-1}^{\prime}}\left(F_{k}^{\prime}\right)=p r_{F_{k-1}^{\prime}}\left(F_{k}\right)$, the chamber $C^{\prime}$ which corresponds to $\beta$ belongs to the same chamber of the arrangement $A_{F_{k-1}^{\prime}}$ as the chosen chamber $C_{0}$. Also $C^{\prime} \prec F_{k-1}^{\prime}$ so that Lemma 2.2.4 implies that $\beta \in \mathbf{W}^{\Gamma_{k-1}^{\prime}}$, as desired.

Assume now we are given $\tau \in \Gamma_{i}$ and $\beta \in \mathbf{W}_{\Gamma_{i}}^{\Gamma_{i}-\{\tau\}}$. By our hypotheses, in order to show the existence of the cell

$$
\begin{aligned}
e^{\prime} & =e\left(\gamma \beta, \Gamma_{1}, \ldots, \Gamma_{i-1}, \Gamma_{i}-\{\tau\}, \beta^{-1} \Gamma_{i+1} \beta, \ldots, \beta^{-1} \Gamma_{d} \beta, \emptyset\right) \\
& =e\left(\gamma \beta, \Gamma_{1}^{\prime}, \ldots, \Gamma_{d}^{\prime}, \emptyset\right),
\end{aligned}
$$

we need to show (prop. 2.3.1) that $\Gamma_{k}^{\prime} \supset \Gamma_{k+1}^{\prime}$ for each $k=1 \ldots, d-1$. This is trivially satisfied for $k<i$. If $k=i$, we have by B) and $\mathrm{C}$ ),

$$
\Gamma_{i+1}^{\prime}=\beta^{-1} \Gamma_{i+1} \beta \subset \Gamma_{i}^{\prime} .
$$

For $k>i$, we have,

$$
\Gamma_{k+1}^{\prime}=\beta^{-1} \Gamma_{k+1} \beta \subset \beta^{-1} \Gamma_{k} \beta=\Gamma_{k}^{\prime} .
$$

Now in order to show that $e^{\prime} \subset \partial e$, we need to see (lemma 1.4.3) that if

$$
(\gamma, \Gamma) \leftrightarrow F=\left(F_{1} \succ \ldots \succ F_{d}\right), \quad\left(\gamma^{\prime}, \Gamma^{\prime}\right) \leftrightarrow F^{\prime}=\left(F_{1}^{\prime} \succ \ldots \succ F_{d}^{\prime}\right),
$$

then

$$
c l\left(p r_{F_{k-1}^{\prime}}\left(F_{k}^{\prime}\right)\right) \supset p r_{F_{k-1}^{\prime}}\left(F_{k}\right), \quad k=1, \ldots, d,
$$

where as above, we set to simplify notations, $F_{0}=0$. The claim is trivial for $k<i$. If $k=i, \Gamma_{i}^{\prime} \subset \Gamma_{i}$ and $\beta \in \mathbf{W}_{\Gamma_{i}}$ imply that $F_{i}^{\prime} \prec F_{i}$. Since $F_{i-1}^{\prime}=F_{i-1}$ this gives our claim. For $k>i$, using $\mathrm{C}$ ) we get that $\left|F_{k}^{\prime}\right|=\left|F_{k}\right|$, while $\beta \in \mathbf{W}^{\Gamma_{k-1}^{\prime}}$ gives that the chambers $C, C^{\prime}$ which correspond respectively to $\gamma, \gamma \beta$ belong to the same chamber of the arrangement $A_{F_{k-1}^{\prime}}$. These two facts clearly give that

$$
c l\left(p r_{F_{k-1}^{\prime}}\left(F_{k}^{\prime}\right)\right) \supset p r_{F_{k-1}^{\prime}}\left(F_{k}\right) \text {. }
$$


Recall that in order to give a $W$ orientation it suffices to orient the cells $e(1, \Gamma \supset \emptyset)$ or equivalently the cells $e(1, \Gamma)$ in $Q^{(d)}$. Such an orientation will be induced by a total ordering, which we fix once and for all, of $S: s_{1}, \ldots, s_{N}$. Let us denote by $H_{s}$ the wall of $C_{0}$ associated to the reflection $s$ and let $f_{s} \in\left(\mathbb{R}^{N}\right)^{*}$ be such that $H_{s}=\operatorname{ker}\left(f_{s}\right)$ and $f_{s}\left(C_{0}\right)<0$. Let $f_{s}^{(k)} \in\left(\left(M_{d}\right)^{N}\right)^{*}$ be defined by

$$
f_{s}^{(k)}\left(X_{1}+\cdots+X_{d}\right)=f_{s}\left(X_{k}\right), \quad k=1, \ldots, d .
$$

Remark 3.1.4. If $\Gamma=\left(\Gamma_{1} \supset \cdots \supset \Gamma_{d}\right)$ the $|\Gamma|$-form

$$
\omega_{\Gamma}:=\bigwedge_{k=1}^{d} \bigwedge_{s \in \Gamma_{k}} d\left(f_{s}^{(k)}\right)
$$

gives an orientation to $F_{\Gamma}^{\perp}$ and therefore to $e(1, \Gamma)$. The product is done according to the ordering of $S$. A cell $e(\gamma, \Gamma)=\gamma \cdot e(1, \Gamma)$ is oriented through the $|\Gamma|-$ form

$$
\left(\gamma^{-1}\right)^{*}\left(\omega_{\Gamma}\right)
$$

so that $\gamma$ preserves the orientation.

Proposition 3.1.5. Let $e:=e(1, \Gamma), \Gamma=\left(\Gamma_{1} \supset \cdots \supset \Gamma_{d}\right)$ and $e^{\prime} \subset \partial(e)$, with

$$
\begin{gathered}
e^{\prime}=e\left(\beta, \Gamma_{1}, \ldots, \Gamma_{i-1}, \Gamma_{i} \backslash\{\tau\}, \beta^{-1} \Gamma_{i+1} \beta, \ldots, \beta^{-1} \Gamma_{d} \beta\right) \\
\beta \in \mathbf{W}_{\Gamma_{i}}^{\Gamma_{i} \backslash\{\tau\}}, \quad \beta^{-1} \Gamma_{i+1} \beta \subset \Gamma_{i} \backslash\{\tau\},
\end{gathered}
$$

be as in Theorem 3.1.3. Then the incidence number $\left[e: e^{\prime}\right]$ equals

$$
(-1)^{i \ell(\beta)+\sum_{k=1}^{i-1}\left|\Gamma_{k}\right|+\mu\left(\Gamma_{i}, \tau\right)+\sum_{k=i+1}^{d} \sigma\left(\beta, \Gamma_{k}\right) .}
$$

Here $\ell$ is the length function with respect to the set $S$ of generators, $\sigma\left(\beta, \Gamma_{k}\right)$ is the number of inversions (in the given ordering of $S$ ) in the map $\Gamma_{k} \rightarrow \beta^{-1} \Gamma_{k} \beta$ and $\mu\left(\Gamma_{i}, \tau\right)$ is the number of reflections in $\Gamma_{i}$ which are less than or equal to $\tau$ in our ordering.

Proof. By remark 3.1.4 one has

$(*) \quad\left[e: e^{\prime}\right]=-\left[\omega_{\Gamma}: d\left(f_{\tau}^{(i)} \circ \beta^{-1}\right) \wedge \bigwedge_{k=1}^{i-1} \bigwedge_{s \in \Gamma_{k}} d\left(f_{s}^{(k)} \circ \beta^{-1}\right) \wedge\right.$

$$
\left.\bigwedge_{s \in \Gamma_{i} \backslash\{\tau\}} d\left(f_{s}^{(i)} \circ \beta^{-1}\right) \wedge \bigwedge_{k=i+1}^{d} \bigwedge_{s \in \beta^{-1} \Gamma_{k} \beta} d\left(f_{s}^{(k)} \circ \beta^{-1}\right)\right] .
$$

Now observe that if $s=\beta^{-1} s^{\prime} \beta$ then $\operatorname{ker}\left(f_{s} \circ \beta^{-1}\right)=\operatorname{ker}\left(f_{s^{\prime}}\right)$ and $f_{s} \circ \beta^{-1}=$ $c f_{s^{\prime}}, c>0$. Moreover, if $\beta=s_{j_{1}} \ldots s_{j_{l}}$ is a reduced expression of $\beta$ then

$$
\bigwedge_{s \in \Gamma_{k}} d\left(f_{s}^{(k)} \circ \beta^{-1}\right)=(-1)^{l} \bigwedge_{s \in \Gamma_{k}} f_{s}^{(k)}
$$


for $k<i$, because $\beta \in \mathbf{W}_{\Gamma_{k}}$. Thus $(*)$ becomes

$$
\begin{aligned}
{\left[e: e^{\prime}\right]=-\left[\omega_{\Gamma}:(-1)^{i \ell(\beta)+\left|\Gamma_{1}\right|+\cdots+\left|\Gamma_{i-1}\right|+\mu\left(\Gamma_{i}, \tau\right)-1} \bigwedge_{k=1}^{i} \bigwedge_{s \in \Gamma_{k}} d f_{s}^{(k)} \wedge\right.} \\
\left.\wedge \bigwedge_{k=i+1}^{d}(-1)^{\sigma\left(\beta, \Gamma_{k}\right)} \bigwedge_{s \in \Gamma_{k}} d f_{s}^{(k)}\right],
\end{aligned}
$$

which concludes the proof.

From now on the exponent appearing in (11) will be denoted by

$$
\alpha(\Gamma, i, \tau, \beta)
$$

It is now clear how to exhibit an algebraic complex of $\mathbb{Z}[\mathbf{W}]$ modules which computes the integer homology of $\mathbf{X}^{(d)}$.

Set $C_{k}^{(d)}$ equal to the free $\mathbb{Z}[\mathbf{W}]$ module with basis the elements $e(\Gamma)$ with $\Gamma \in S_{k}^{(d)}$ (see coroll. 2.3.5). Define a $\mathbb{Z}[\mathbf{W}]$-linear differential $\delta_{k}: C_{k}^{(d)} \rightarrow C_{k-1}^{(d)}$ by

$$
\delta_{k} e(\Gamma)=\sum_{\substack{1 \leq i \leq d \\\left|\Gamma_{i}\right|>\left|\bar{\Gamma}_{i+1}\right|}} \sum_{\tau \in \Gamma_{i}} \sum_{\substack{\beta \in \mathbf{W}_{\Gamma_{i}}^{\Gamma_{i} \backslash\{\tau\}} \\ \beta^{-1} \Gamma_{i+1} \beta \subset \Gamma_{i} \backslash\{\tau\}}}(-1)^{\alpha(\Gamma, i, \tau, \beta)} \beta e\left(\Gamma^{\prime}\right),
$$

where

$$
\Gamma^{\prime}=\left(\Gamma_{1}, \ldots, \Gamma_{i-1}, \Gamma_{i} \backslash\{\tau\}, \beta^{-1} \Gamma_{i+1} \beta, \ldots, \beta^{-1} \Gamma_{d} \beta\right)
$$

Using our previous analysis and the fact that $\mathbf{X}^{(d)}$ is $d-1$ connected we get

Theorem 3.1.6. $H_{*}\left(\mathbf{X}^{(d)} ; \mathbb{Z}\right) \cong H_{*}(C(d) ; \delta)$. In particular, $H_{i}(C(d) ; \delta)=\mathbb{Z}$, the trivial $\mathbb{Z}[\mathbf{W}]$ module, and $H_{i}(C(d) ; \delta)=0$ for $0<i<d$.

Notice that by mapping $e(\Gamma)$ to $e(\Gamma, \emptyset)$ we obtain for each $d$ a map of complexes $C(d) \rightarrow C(d+1)$ which is an isomorphism in degree up to $d$. Taking the limit for $d$ going to infinity we obtain a complex of $\mathbb{Z}[\mathbf{W}]$ modules $(C(\infty), \delta)$ which gives a free resolution of the trivial $\mathbb{Z}[\mathbf{W}]$ module $\mathbb{Z}$. For completeness we give an explicit description of $(C(\infty), \delta)$. Set

$$
S^{(\infty)}:=\left\{\Gamma=\left(\Gamma_{1} \supset \Gamma_{2} \supset \ldots\right): \Gamma_{i}=\emptyset \text { for } i>>0\right\}
$$

and

$$
S_{k}^{(\infty)}:=\left\{\Gamma \in S^{(\infty)}:|\Gamma|=k\right\}, \quad k=0, \ldots, N d .
$$


Then $C_{k}^{(\infty)}$ equals to the free $\mathbb{Z}[\mathbf{W}]$ module with basis the elements $e(\Gamma)$ with $\Gamma \in S_{k}^{(\infty)}$. As for the differential, we have, from (12) and (13), $\delta_{k}: C_{k}^{(\infty)} \rightarrow C_{k-1}^{(\infty)}$ is given by

$$
\delta_{k} e(\Gamma)=\sum_{\substack{i \geq 1 \\\left|\Gamma_{i}\right|>\left|\Gamma_{i+1}\right|}} \sum_{\tau \in \Gamma_{i}} \sum_{\substack{\beta \in \mathbf{W}_{\Gamma_{i}}^{\left.\Gamma_{i} \backslash \backslash \tau\right\}} \\ \beta^{-1} \Gamma_{i+1} \beta \subset \Gamma_{i} \backslash\{\tau\}}}(-1)^{\alpha(\Gamma, i, \tau, \beta)} \beta e\left(\Gamma^{\prime}\right),
$$

where

$$
\Gamma^{\prime}=\left(\Gamma_{1}, \ldots, \Gamma_{i-1}, \Gamma_{i} \backslash\{\tau\}, \beta^{-1} \Gamma_{i+1} \beta, \beta^{-1} \Gamma_{i+2} \beta, \ldots\right) .
$$

Thus we have

Theorem 3.1.7. The complex $(C(\infty), \delta)$ gives a free resolution of the trivial $\mathbb{Z}[\mathbf{W}]$ module $\mathbb{Z}$.

Remark 3.1.8. The above theorem is the algebraic reflection of the fact that $\mathbf{Y}^{(\infty)}=\lim _{d \rightarrow \infty} \mathbf{Y}^{(d+1)}$ is contractible and that $\mathbf{W}$ acts cellularely and freely on $\mathbf{X}^{(\infty)} \cong \mathbf{Y}^{(\infty)}$. So we have

$$
k(\mathbf{W}, 1)=\mathbf{X}_{\mathbf{W}}^{(\infty)} \cong \mathbf{Y}^{(\infty)} / \mathbf{W}
$$

where $\mathbf{X}_{\mathbf{W}}^{(\infty)}=\lim _{d \rightarrow \infty} \mathbf{X}_{\mathbf{W}}^{(d)}$, the inclusion between $\mathbf{X}_{\mathbf{W}}^{(d)}$ and $\mathbf{X}_{\mathbf{W}}^{(d+1)}$ being as in (6).

Remark 3.1.9. By standard facts we obtain that, given a $\mathbb{Z}[\mathbf{W}]$ module $M$, setting $C_{k}(M)=C_{k} \otimes_{\mathbb{Z}[\mathbf{W}]} M$ (resp. $\left(C^{k}(M)=\operatorname{Hom}_{\mathbb{Z}[\mathbf{W}]}\left(C_{k}, M\right)\right)$ and setting $\partial_{k}: C_{k}(M) \rightarrow C_{k+1}(M)$ equal to $\delta_{k} \otimes i d\left(\right.$ resp. $\partial^{k}: C^{k}(M) \rightarrow C^{k+1}(M)$ equal to $\partial^{k}(f)(x)=f\left(\delta_{k+1}(x)\right)$ for each $\left.x \in C_{k}\right)$, we obtain an explicit cochain complex whose homology is $H_{*}(\mathbf{W}, M)$ (resp. whose cohomology is $H^{*}(\mathbf{W}, M)$ ).

\section{The inclusion $\mathbf{X}_{\mathrm{W}}^{(1)} \hookrightarrow \mathbf{X}_{\mathrm{W}}^{(\infty)}$}

4.1. In order to simplify notations we shall set $\mathbf{X}_{\mathbf{W}}=\mathbf{X}_{\mathbf{W}}^{(1)}$. This is the same space as considered in [S1], and it is $k\left(G_{\mathbf{W}}, 1\right)$, where $G_{\mathbf{W}}$ is the Artin group associated to $\mathbf{W}$. By (6), $\mathbf{X}_{\mathbf{W}}$ can be identified with the subcomplex of $\mathbf{X}_{\mathbf{W}}^{(\infty)}$ given by the cells $\Gamma=\left(\Gamma_{1} \supset \emptyset \supset \ldots\right)$. We could also drop the "empty" part of $\Gamma$.

Consider now a $\mathbb{Z}[\mathbf{W}]$ module $M$ and take the complexes $C^{*}(M)$ considered in 3.1.9 and $C^{k}(1)(M)=\operatorname{Hom}_{\mathbb{Z}[\mathbf{W}]}\left(C_{k}(1), M\right)$. Clearly the cohomology of $C^{k}(1)(M)$ equals $H^{*}\left(G_{\mathbf{W}}, M\right)$, where $M$ is considered as a $\mathbb{Z}\left[G_{\mathbf{W}}\right]$-module via the quotient $\pi: G_{\mathbf{W}} \rightarrow \mathbf{W}$. Also the map $C^{*}(M) \rightarrow C^{*}(1)(M)$ induced by the inclusion $C^{*}(1) \rightarrow C^{*}(\infty)$ gives in cohomology the homomorphism $\pi^{*}$. 
Looking at degree $n$ (we assume that $\mathbf{W}$ acts essentially on $\mathbb{R}^{n}$ ), we get:

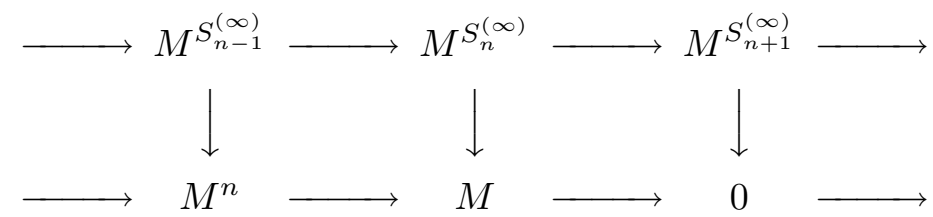

Let us consider functions $f \in M^{S_{n}^{(\infty)}}$ such that $f\left(\Gamma=\left(\Gamma_{1} \supset \Gamma_{2} \supset \ldots\right)\right)=0$, if $\left|\Gamma_{i}\right|>0$ for some $i>1$ (equivalently, if $\left|\Gamma_{2}\right|>0$ ). Let us compute the coboundary of $f$.

(a) $\delta^{n}(f)\left(\Gamma_{1} \supset \Gamma_{2} \supset \ldots\right)=0$ if $\left|\Gamma_{2}\right|>1$ or $\left|\Gamma_{3}\right|>0$.

In fact, by formula (18) we get a linear combination of $f$ computed on chains with $\left|\Gamma_{2}\right|>0$.

(b) If $\Gamma_{1}=\left\{s_{j_{1}}<\cdots<s_{j_{n}}\right\}, \Gamma_{2}=\left\{s_{j_{k}}\right\}$ then

$$
\begin{aligned}
\delta^{n}(f)\left(\Gamma_{1} \supset \Gamma_{2}\right) & =\sum_{\beta_{2} \in \mathbf{W}_{s_{j_{k}}}}(-1)^{\alpha\left(\Gamma, 2,1, \beta_{2}\right)} \rho\left(\beta_{2}\right) \cdot f\left(\Gamma_{1}\right) \\
& =(-1)^{n+1} f\left(\Gamma_{1}\right)+(-1)^{n+1} \rho\left(s_{j_{k}}\right) \cdot f\left(\Gamma_{1}\right) \\
& =(-1)^{n+1}\left(1+\rho\left(s_{j_{r}}\right)\right) \cdot f\left(\Gamma_{1}\right) .
\end{aligned}
$$

Proposition 4.1.1. Let $M=\mathbb{Z}[-1]$, be the sign module. Then, the map $\pi^{*}: H^{n}(\mathbf{W}, \mathbb{Z}[-1]) \rightarrow H^{n}\left(G_{\mathbf{W}}, \mathbb{Z}[-1]\right)$ is surjective.

Proof. It is an immediate consequence of diagram (16) and formula (17).

4.2. Recall now that the Schwartz genus of a fibration is defined as the minimal cardinality of an open covering of the base space, such that there exists a section over each open set.

From the previous proposition we can deduce the Schwartz genus of the covering $p: \mathbf{X}=\mathbf{X}^{(1)} \rightarrow \mathbf{X}_{\mathbf{W}}$.

\section{Theorem 4.2.1.}

(a) For all irreducible Coxeter groups $\mathbf{W}$, except the case $A_{n}$, the covering $p: \mathbf{X} \rightarrow \mathbf{X}_{\mathbf{W}}$ has Schwartz genus $g(p)=n+1$, $n$ being the rank of $\mathbf{W}$.

(b) In case $A_{n} g(p)=n+1$, if $n+1$ is a power of a prime number.

Proof. For all $\mathbf{W}$ one has $g(p) \leq n+1$, since $\mathbf{X}_{\mathbf{W}}$ is an $n$ dimensional $C W$-complex.

The converse inequality is obtained from $g(p) \geq h(p)$ because $h(p)$ is the homological genus of $p$ (see [Va; ch.2, §1]). By using computations in [D-S-S] we obtain that for all $\mathbf{W} \neq A_{n} H^{n}\left(\mathbf{X}_{\mathbf{W}}, \mathbb{Z}[-1]\right) \neq 0$. From this using proposition 4.1.1 we obtain part (a).

Part (b) is analogous: from $[\mathrm{D}-\mathrm{S}-\mathrm{S}]$ we have $H^{n}\left(\mathbf{X}_{\mathbf{W}}, \mathbb{Z}[-1]\right)=\mathbb{Z} /\left(\varphi_{n+1}(1)\right)$, where $\varphi_{k}(q)$ is the $k$ th-cyclotomic polynomial. But it is well-known $\varphi_{k}(1) \neq 1$ iff $k$ is a power of a prime number. Therefore part (b) follows. 


\section{Examples}

To finish we give here some examples where we used the algebraic complex in part 3 to make computations. Given a partition of $n,\left[1^{i_{1}}, 2^{i_{2}}, \ldots, n^{i_{n}}\right]$ with $\sum_{h} i_{h} h=n$, we denote by the same letter the corresponding Specht module over $\mathbb{Z}($ see $[J])$.

Theorem 5.1. One has:

$$
\begin{aligned}
& H^{k}\left(A_{2} ;[3]\right)= \begin{cases}\mathbb{Z} / 6 \mathbb{Z} & \text { if } k \equiv 0(\bmod 4), k>0, \\
\mathbb{Z} / 2 \mathbb{Z} & \text { if } k \equiv 2(\bmod 4), \\
0 & \text { if } 2 \nmid k, \\
\mathbb{Z} & \text { if } k=0 .\end{cases} \\
& \left.H^{k}\left(A_{2} ; 1^{3}\right]\right)= \begin{cases}0 & \text { if } k \equiv 0(\bmod 4), \\
\mathbb{Z} / 3 \mathbb{Z} & \text { if } k \equiv 2(\bmod 4), \\
\mathbb{Z} / 2 \mathbb{Z} & \text { if } 2 \nmid k .\end{cases} \\
& H^{k}\left(A_{2} ;[2,1]\right)= \begin{cases}\mathbb{Z} / 3 \mathbb{Z} & \text { if } k \equiv 1(\bmod 4), \\
0 & \text { otherwise. }\end{cases} \\
& H^{k}\left(A_{3},[4]\right)= \begin{cases}\left(\frac{\mathbb{Z}}{2 \mathbb{Z}}\right)^{\left[\frac{k}{3}\right]} \times \frac{\mathbb{Z}}{12 \mathbb{Z}} & \text { if } k \equiv 0 \bmod (4), k>0 \\
\left(\frac{\mathbb{Z}}{2 \mathbb{Z}}\right)^{\left[\frac{k}{3}\right]+1} & \text { if } k \equiv 2 \bmod (4) \\
\left(\frac{\mathbb{Z}}{2 \mathbb{Z}}\right)^{\left[\frac{k}{3}\right]} & \text { if } 2 \nmid k \\
\mathbb{Z} & \text { if } k=0\end{cases} \\
& H^{k}\left(A_{3},\left[1^{4}\right]\right)= \begin{cases}\left(\frac{\mathbb{Z}}{2 \mathbb{Z}}\right)^{\left[\frac{k}{3}\right]} \times \frac{\mathbb{Z}}{3 \mathbb{Z}} & \text { if } k \equiv 2 \bmod (4) \\
\left(\frac{\mathbb{Z}}{2 \mathbb{Z}}\right)^{\left[\frac{k}{3}\right]} & \text { if } k \equiv 0 \bmod (4) \\
\left(\frac{\mathbb{Z}}{2 \mathbb{Z}}\right)^{\left[\frac{k}{3}\right]+1} & \text { if } 2 \nmid k\end{cases} \\
& H^{k}\left(A_{3},[3,1]\right)= \begin{cases}\left(\frac{\mathbb{Z}}{2 \mathbb{Z}}\right)^{\left[\frac{k-1}{3}\right]} \times \frac{\mathbb{Z}}{4 \mathbb{Z}} & \text { if } k \equiv 1 \bmod (4) \\
\left(\frac{\mathbb{Z}}{2 \mathbb{Z}}\right)^{\left[\frac{k+2}{3}\right]} & \text { otherwise }\end{cases} \\
& H^{k}\left(A_{3},\left[2,1^{2}\right]\right)=\left(\frac{\mathbb{Z}}{2 \mathbb{Z}}\right)^{\left[\frac{k+1}{3}\right]} \\
& H^{k}\left(A_{3},[2,2]\right)= \begin{cases}\left(\frac{\mathbb{Z}}{2 \mathbb{Z}}\right)^{\left[\frac{k+1}{6}\right]} \times \frac{\mathbb{Z}}{3 \mathbb{Z}} & \text { if } k \equiv 1 \bmod (4) \\
\left(\frac{\mathbb{Z}}{2 \mathbb{Z}}\right)^{\left[\frac{k+1}{6}\right]} & \text { if } k \equiv 3 \bmod (4) \\
\left(\frac{\mathbb{Z}}{2 \mathbb{Z}}\right)^{\left[\frac{k+4}{6}\right]} & \text { otherwise }\end{cases}
\end{aligned}
$$

The cohomology of $A_{2}$ is known (at least with trivial coefficients) and is periodic.

Case $A_{3}$ seems to be new (see also [B-C] for related computations). 


\section{References}

[B-C] D.J. Benson, and F.R. Cohen, Mapping class group of low genus and their cohomology, Mem. Amer. Math. Soc. 90 (1991).

[B-Z] A. Bjorner and G.M. Ziegler, Combinatorial stratification of complex arrangements, J. Amer. Math. Soc. 5 (1992), 105-149.

[Bou] N. Bourbaki, Groupes et algebres de Lie (chap. 4,5,6), Masson, Paris, 1981.

[Br] E. Brieskorn, Sur les groupes de tresses, Sem. Bourbaki, Lec. Notes in Math., vol. 317, Springer, Berlin, 1973, pp. 21-44.

[D-S] C. De Concini and M. Salvetti, Cohomology of Artin groups, Math. Res. Lett. 3 (1996), 293-297.

[D-S-S] C. De Concini, M. Salvetti, and F. Stumbo, The top cohomology of Artin groups with coefficients in rank 1 local systems over $\mathbb{Z}$, Topology Appl. 78 (1997), 5-20.

[De] P. Deligne, Les immeubles des groupes de tresses generalizes, Invent. Math. 17 (1972), 273-302.

[G-R] I.M. Gelfand and L. Rybnykov, Algebraic and topological invariants of oriented matroids, Dokladi 307 (1989), 791-795.

[Hum] J.E. Humphreys, Reflection groups and Coxeter groups, Cambridge Studies in Advanced Mathematics, 29, Cambridge University Press, Cambridge, 1992.

[J] G.D. James, The representation theory of the symmetric groups, Lecture Notes in Mathematics, 682, Springer, Berlin, 1978.

[S0] M. Salvetti, Topology of the complement of real hyperplanes in $\mathbb{C}^{n}$, Invent. Math. 88 (1987), 167-189.

[S1] M. Salvetti, The homotopy type of Artin groups, Math. Res. Lett. 1 (1994), 565-577.

[Sw] R. Swan, Periodic resolutions for finite groups., Ann. of Math. (2) 72 (1960), 267-291.

[Va] V.A. Vassiliev, Complements of discriminants of smooth maps: topology and applications, Translations of Mathematical Monographs, 98, American Mathematical Society, Providence, RI, 1992.

Dipartimento di Matematica "G. Castelnuovo", Universita' di Roma "La Sapienza"

E-mail address: deconcin@mat.uniroma1.it

Dipartimento di Matematica "L. Tonelli", Universita' di Pisa

E-mail address: salvetti@paley.dm.unipi.it 